\title{
Minimax design of two-channel nonuniform-division FIR filter banks
}

\author{
J.-H.Lee \\ D.-C.Tang
}

Indexing terms: Filters and filtering, Optimisation, Two-channel nomuniform-division, FIR filter banks, Algorithms

\begin{abstract}
The paper deals with the minimax design of two-channel nonuniform-division filter (NDF) banks. Based on a linearisation scheme, the design problem is formulated as an optimisation problem with linear constraints. The authors present a method to design a two-channel NDF bank using a modified dual-affine scaling variant of Karmarkar's algorithm. This method provides the optimal results that the linear-phase FIR analysis and synthesis filters have equiripple stopband response and the resulting NDF bank also shows equiripple reconstruction error behaviour. The effectiveness of the proposed design technique is demonstrated by several simulation examples.
\end{abstract}

\section{Introduction}

In many areas, such as the subband coding of speech signals [1], communication systems [2] and short-time spectral analysis [3], quadrature mirror filter (QMF) banks find a very important role. Recently, their usefulness has been extended to the area of image subband coding [4], which has been recognised as an effective technique for high quality image coding at low bit rates. In these applications, a QMF bank is used to decompose a signal into subbands with equal bandwidth and the subband signals in the analysis system are decimated by an integer which is equal to the number of the subbands. However, uniform-subband decomposition is not an appropriate scheme to match the requirements in a great variety of applications. An important example is critical band analysis with a filter bank which can be utilised in spectral analysis, coding, enhancement, speech recognition and audio signals. For the subband coding of speech and audio signals, the most appropriate decomposition must consider the critical bands of the ear. It has been considered in [5] that these critical bands have nonuniform bandwidths and cannot be easily constructed by the conventional tree structure based on two-channel QMF banks. Thus, it is worth exploiting the design problem of nonuniform-division filter (NDF) banks.

\section{(C) IEE, 1998}

IEE Proceedings online no. 19981860

Paper first received 26th August and in revised form 18th December 1997 The authors are with the Department of Electrical Engineering, Room 517, Building 2, National Taiwan University, Taipei 106, Taiwan
The basic theory regarding the principle and the related conditions of perfect reconstruction for NDF banks has been presented in [6]. Methods for designing the NDF banks were also proposed in [6]. However, it is difficult to solve the resulting design problem with nonlinear constraints. In [7], a structure for NDF banks was introduced and a design method based on the use of pseudo-QMF was presented. The main drawback is that FIR filters with complex coefficients are required by the resulting NDF bank to reduce the aliasing distortion. Recently, one of the authors considered a structure for two-channel NDF banks and proposed design methods for optimally designing NDF banks based on the least-absolute error criteria in [8].

In this paper, NDF banks with structures similar to [8], as shown in Fig. 1, are considered. We deal with the minimax design of a two-channel NDF bank. A method for designing NDF banks with equiripple reconstruction error and equiripple stopband response for its linear-phase (LP) FIR analysis and synthesis filters is developed. A modified dual-affine scaling (MDAS) variant of Karmarkar's algorithm of [9], in conjunction with a linearisation scheme, is presented, to solve the resulting nonlinear design problem. It is shown that the optimal coefficients for the LP FIR analysis and synthesis filters can be found through solving only linear equations. Simulation results show that very satisfactory NDF banks can be obtained using the proposed technique.
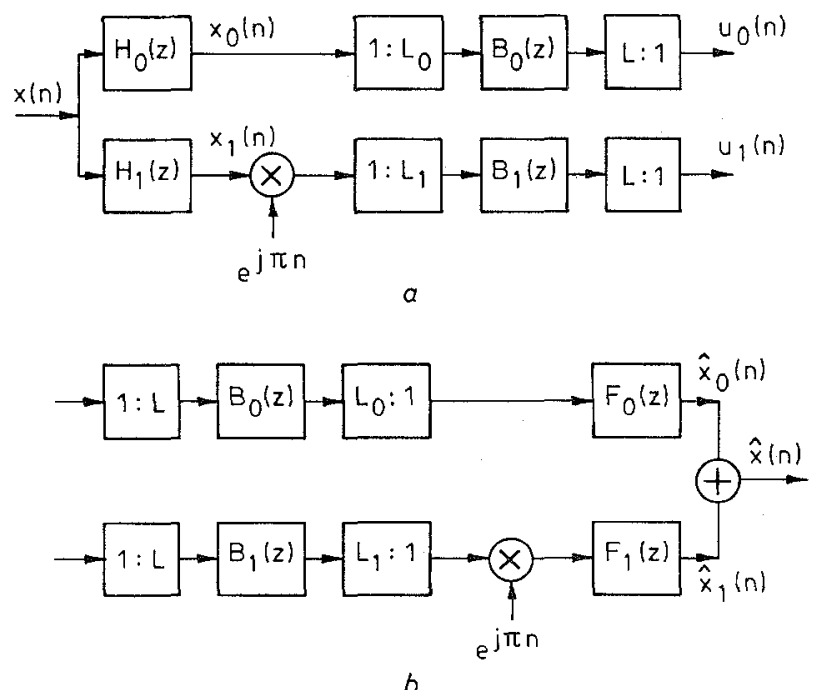

Fig.1 Two-channel nonuniform-division maximally decimated filter bank system

a Analysis system

$b$ Synthesis system 


\section{Two-channel nonuniform-division FIR filter \\ banks}

Consider the two-channel nonuniform-division filter (NDF) bank with the architecture given in [8] which is shown in Fig. 1. The linear-phase (LP) analysis lowpass and highpass filters are designated by $H_{0}(z)$ and $H_{1}(z)$, respectively, while the LP synthesis lowpass and highpass filters are designated by $F_{0}(z)$ and $F_{1}(z)$, respectively. $B_{0}(z)$ and $B_{1}(z)$ are two lowpass filters responsible for achieving aliasing-free operation during the rational decimation and interpolation. It can be shown that using the modulations of multiplying $\exp (j n \pi)$ in highpass subband channel leads to the favourable result that $B_{1}(z)$ can be a lowpass filter with real coefficients. The desired magnitude responses for the analysis filters $H_{0}(z)$ and $H_{1}(z)$ with passband widths equal to $L_{0} \pi / L$ and $L_{1} \pi / L$, respectively, are shown in Fig. 2, where $L=L_{0}+L_{1} \cdot \omega_{p}$ and $\omega_{s}$ denote the related band-edge frequencies.

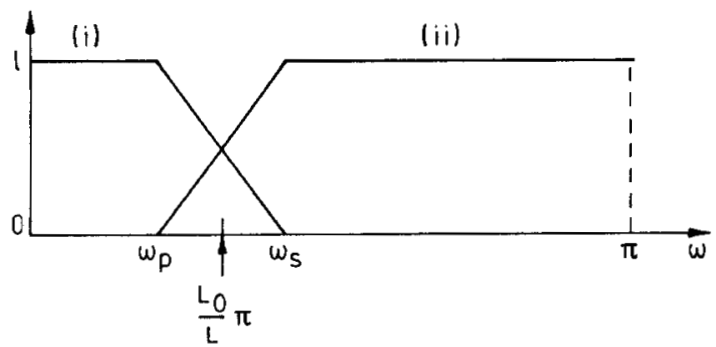

Fig. 2 Desired magnitude specifications for the analysis filters (i) $H_{0}(\omega) / \sqrt{ }\left(L L_{0}\right) ;\left(\right.$ ii) $H_{1}(\omega) / \sqrt{ }\left(L L_{1}\right)$

Following eqns. 45, 46 and 47 obtained from the Appendix we can reformulate the conditions required for perfect reconstruction as follows:

$$
\begin{aligned}
& T(\omega)=1, \quad \text { for } 0 \leq \omega \leq \pi \\
& H_{0}(\omega)=0, \quad \text { for } \omega_{s} \leq \omega \leq \pi \\
& H_{1}(\omega)=0, \quad \text { for } 0 \leq \omega \leq \omega_{p} \\
& \frac{1}{\sqrt{L L_{0}}} H_{0}(\omega)=\frac{1}{\sqrt{L L_{1}}} H_{1}\left(\omega_{p}+\omega_{s}-\omega\right), \\
& \text { for } \omega_{p} \leq \omega \leq \omega_{s}
\end{aligned}
$$

Eqns. 1 reveal that the conditions for perfect reconstruction can be met only when $H_{0}(z)$ and $H_{1}(z)$ have infinite filter length. Therefore, the design problem of the two-channel NDF banks of Fig. 1 is finding such $H_{0}(z)$ and $H_{1}(z)$ with finite filter length that the conditions listed in eqn. 1 can be approximately met in some optimal sense.

\section{Optimal design of two-channel NDF banks in the minimax sense}

\subsection{Problem formulation using a linearisafion scheme}

For the case of optimally designing two-channel NDF banks in the minimax sense, both of the designed filters $H_{0}(z)$ and $H_{1}(z)$ have equiripple stopband response, while the resulting NDF bank shows equiripple reconstruction error behaviour. From eqn. 1, let the design specifications for the magnitude responses of $H_{0}(z)$ and $H_{1}(z)$ be given as follows:

$$
\frac{H_{0}(\omega)}{\sqrt{L L_{0}}}=\left\{\begin{array}{l}
D_{0}(\omega)=1 \text { with } W_{m x}(\omega)=1 \\
\quad \text { for } 0 \leq \omega \leq \omega_{p} \\
D_{0}(\omega)=0 \text { with } W_{m x}(\omega)=r \\
\text { for } \omega_{s} \leq \omega \leq \pi
\end{array}\right.
$$

and

$$
\frac{H_{1}(\omega)}{\sqrt{L L_{1}}}=\left\{\begin{array}{l}
D_{1}(\omega)=0 \text { with } W_{m x}(\omega)=r \\
\text { for } 0 \leq \omega \leq \omega_{p} \\
D_{1}(\omega)=1 \text { with } W_{m x}(\omega)=1 \\
\text { for } \omega_{s} \leq \omega \leq \pi
\end{array}\right.
$$

respectively, where $D_{i}(\omega)$ denote the desired magnitude responses, $W_{m x}(\omega)$ the minimax weightmg function, and $r$ the ratio between the passband and stopband ripples. Assume that the designed FIR filters $H_{0}(z) / \sqrt{ }\left(L L_{0}\right)$ and $H_{1}(z) / \sqrt{ }\left(L L_{1}\right)$ have peak error $\delta$ in their passbands. It can be shown from eqn. 45 that the corresponding magnitude response $T(\omega)$ has the following behaviour:

$$
\begin{aligned}
& (1-\delta)^{2} \leq T(\omega) \leq(1+\delta)^{2}+\left(\frac{\delta}{r}\right)^{2} \\
& \text { for } \omega \in\left[0, \omega_{p}\right] \cup\left[\omega_{s}, \pi\right]
\end{aligned}
$$

Eqn. 4 reveals that $T(\omega)$ is not constrained over the frequency range of $\left(\omega_{p}, \omega_{s}\right)$. Hence, we have to impose the following additional condition for $T(\omega)$ :

$$
(1-\delta)^{2} \leq T(\omega) \leq(1+\delta)^{2}, \quad \text { for } \omega \in\left(\omega_{p}, \omega_{s}\right)
$$

to ensure that $T(\omega)$ shows the equiripple response over the entire frequency range. However, we note that the required constraint of eqn. 5 is a nonlinear function of the filter coefficients $h_{0}(n)$ and $h_{1}(n)$, and, hence, results in a highly nonlinear optimisation problem. To overcome this difficulty, we propose an appropriate linearisation scheme for dealing with eqn. 5. Consider the following constraint:

$$
\begin{aligned}
(1-\delta) & \leq \frac{\frac{1}{L L_{0}} H_{0}(\omega) \tilde{H}_{0}(\omega)+\frac{1}{L L_{1}} H_{1}(\omega) \tilde{H}_{1}(\omega)}{\sqrt{\tilde{T}}(\omega)} \\
& \leq(1+\delta), \quad \text { for } \omega \in\left(\omega_{p}, \omega_{s}\right)
\end{aligned}
$$

where

$$
\tilde{T}(\omega)=\frac{1}{L L_{0}} \tilde{H}_{0}^{2}(\omega)+\frac{1}{L L_{1}} \tilde{H}_{1}^{2}(\omega)
$$

denotes the magnitude response of the designed NDF bank corresponding to the designed LP FIR analysis filters $\tilde{H}_{0}(z)$ and $\tilde{H}_{1}(z)$ whose magnitude responses are given by $\tilde{H}_{0}(\omega)$ and $\tilde{H}_{1}(\omega)$, respectively. Note that the constraint of eqn. 6 is equivalent to the required constraint of eqn. 5 when $\tilde{H}_{0}(\omega)=H_{0}(\omega)$ and $\tilde{H}_{1}(\omega)=$ $H_{1}(\omega)$. Therefore, eqn. 7 becomes a linear constraint, if $\tilde{H}_{0}(\omega)$ and $\tilde{H}_{1}(\omega)$ are set to the magnitude responses of the obtained LP FIR analysis filters during the design process.

Next, let $\mathbf{V}$ and $\tilde{\mathbf{V}}$ be two vectors given by

$$
\mathbf{V}=\left[\frac{H_{0}(\omega)}{\sqrt{L L_{0}}}, \frac{H_{1}(\omega)}{\sqrt{L L_{1}}}\right]^{T}
$$

and

$$
\tilde{\mathbf{V}}=\left[\frac{\tilde{H}_{0}(\omega)}{\sqrt{L L_{0}}}, \frac{\tilde{H}_{1}(\omega)}{\sqrt{L L_{1}}}\right]^{T}
$$

Substituting eqns. 7 and 8 into eqn. 6 yields

$$
(1-\delta)^{2} \leq \frac{\langle\mathbf{V} \cdot \tilde{\mathbf{V}}\rangle^{2}}{\langle\tilde{\mathbf{V}} \cdot \tilde{\mathbf{V}}\rangle} \leq(1+\delta)^{2}, \quad \text { for } \omega \in\left(\omega_{p}, \omega_{s}\right)
$$

where $\langle\cdot\rangle$ denotes the operation of vector inner product. From Cauchy-Schwartz inequality, it follows that

$$
\langle\tilde{\mathbf{V}} \cdot \tilde{\mathbf{V}}\rangle \cdot\langle\mathbf{V} \cdot \mathbf{V}\rangle \geq\langle\mathbf{V} \cdot \tilde{\mathbf{V}}\rangle^{2}
$$


From eqns. 9 and 10 , we obtain

$$
T(\omega)=\langle\mathbf{V} \cdot \mathbf{V}\rangle \geq(1-\delta)^{2}, \quad \text { for } \omega \in\left(\omega_{p}, \omega_{s}\right)
$$

Eqn. 11 reveals that the inequality holds if $H_{0}(z)$ and $H_{1}(z)$ satisfy the constraint shown by eqn. 6 for the obtained $\tilde{\mathbf{V}}$ during the design process. Moreover, if $\tilde{\mathbf{V}}$ is very close to $\mathbf{V}$ for $\omega \in\left(\omega_{p}, \omega_{s}\right)$, we then have

$$
\begin{aligned}
& (1+\delta)^{2} \geq \frac{\langle\mathbf{V} \cdot \tilde{\mathbf{V}}\rangle^{2}}{\langle\tilde{\mathbf{V}} \cdot \tilde{\mathbf{V}}\rangle} \approx\langle\mathbf{V} \cdot \mathbf{V}\rangle=T(\omega), \\
& \text { for } \omega \in\left(\omega_{p}, \omega_{s}\right)
\end{aligned}
$$

Hence, the constraint shown by eqn. 5 could be met during the design process.

Based on these results, we formulate the problem for the minimax design of two-channel NDF banks as follows:

\section{minimise $\delta$}

subject to

$$
\left\{\begin{aligned}
1-\delta & \leq \frac{H_{0}(\omega)}{\sqrt{L L_{0}}} \leq 1+\delta, \quad \text { for } \omega \in\left[0, \omega_{p}\right] \\
-\frac{\delta}{r} & \leq \frac{H_{1}(\omega)}{\sqrt{L L_{1}}} \leq \frac{\delta}{r}, \quad \text { for } \omega \in\left[0, \omega_{p}\right] \\
1-\delta & \leq \frac{H_{1}(\omega)}{\sqrt{L L_{1}}} \leq 1+\delta, \quad \text { for } \omega \in\left[\omega_{s}, \pi\right] \\
-\frac{\delta}{r} & \leq \frac{H_{0}(\omega)}{\sqrt{L L_{0}}} \leq \frac{\delta}{r}, \quad \text { for } \omega \in\left[\omega_{s}, \pi\right] \\
1-\delta & \leq \frac{\frac{1}{L L_{0}} H_{0}(\omega) \tilde{H_{0}}(\omega)+\frac{1}{L L_{1}} H_{1}(\omega) \tilde{H}_{1}(\omega)}{\sqrt{\tilde{T}(\omega)}} \\
& \leq 1+\delta, \quad \text { for } \omega \in\left(\omega_{p}, \omega_{s}\right) \\
-\frac{\delta}{r} & \leq \frac{H_{0}(\omega)}{\sqrt{L L_{0}}}-\frac{H_{1}(\omega)}{\sqrt{L L_{1}}} \leq \frac{\delta}{r}, \quad \text { for } \omega \in\left[\omega_{p}, \omega_{s}\right]
\end{aligned}\right.
$$

Examining the constraints listed in eqn. 13, we note that the first five constraints are used to ensure that the resulting $T(\omega)$ satisfies the required constraints shown by eqns. 4 and 5 . The second and fourth constraints are used to ensure that the designed $H_{0}(z)$ and $H_{1}(z)$ have the minimax stopband response, while the last constraint is employed to guarantee that the required aliasing cancellation can be met. Moreover, all of the constraints listed in eqn. 13 are linear functions of the filter coefficients $h_{0}(n)$ and $h_{1}(n)$. Therefore, the design problem shown by eqn. 13 is an optimisation problem with linear constraints. Accordingly, the overall design task is to find the filter coefficients $h_{0}(n)$ and $h_{1}(n)$ such that the peak ripple $\delta$ is minimised.

Let $\left\{\omega_{1}=0, \omega_{2}, \ldots, \omega_{I}=\omega_{p}, \ldots, \omega_{J}=\omega_{s}, \ldots, \omega_{K}=\pi\right\}$ be a dense grid of frequencies, linearly distributed in the range of $\omega=0$ to $\omega=\pi$, for evaluating the magnitude response of the NDF bank and the related error functions defined in the preceding text. Assume that $H_{0}(z)$ is a case 2 LP FIR filter. To formulate the considered design problem in a more compact form, the expressions given in eqns. 42 and 43 , for $H_{0}(\omega)$ and $H_{1}(\omega)$, are substituted into the linear constraints shown by eqn. 13. Then, we put the related filter coefficients, the cosine and sine terms into matrices as shown in Section 3.1.1, where $\mathbf{U}_{0}$ is a $K \times N_{0} / 2$ matrix containing the related cosine terms for $\omega \in[0, \pi]$. $\mathbf{U}_{1}$ is a $K \times N_{1} / 2$ matrix containing the related sine terms for $\omega \in[0, \pi]$. $\mathrm{U}_{0 p}$ is an $I \times N_{0} / 2$ matrix containing the related cosine terms for $\omega \in\left[0, \omega_{p}\right] . \mathbf{U}_{1 p}$ is a $(K-J+1) \times N_{1} / 2$ matrix containing the related sine terms for $\omega \in\left[\omega_{s}, \pi\right] . \mathbf{U}_{0 s}$ is a $(K-J+1) \times N_{0} / 2$ matrix containing the related cosine terms for $\omega \in\left[\omega_{s}, \pi\right]$. $\mathbf{U}_{1, s}$ is an $I \times N_{1} / 2$ matrix containing the related sine terms for $\omega \in\left[0, \omega_{p}\right] . \mathbf{U}_{0 t}$ is a $(J-I+1) \times N_{0} / 2$ matrix containing the related cosine terms for $\omega \in\left[\omega_{p}, \omega_{s}\right] . \mathbf{U}_{1 t}$ is a $(J-I+1) \times N_{1} / 2$ matrix containing the related sine terms for $\omega \in\left[\omega_{p}, \omega_{s}\right] . \mathbf{U}_{a t}$ is a $(J-I+1) \times N_{0} / 2$ matrix containing the weighted cosine terms for $\omega \in\left[\omega_{p}, \omega_{s}\right] . \mathbf{U}_{b t}$ is a $(J-I+1) \times N_{1} / 2$ matrix containing the weighted sine terms for $\omega \in\left[\omega_{p}\right.$, $\omega_{s}$ ]. Let $\mathbf{y}$ and $\mathbf{z}$ be two vectors containing the independent filter coefficients as follows:

$$
\mathbf{y}=\left[h_{0}(0), h_{0}(1), \ldots, h_{0}\left(\frac{N_{0}}{2}-1\right)\right]^{T}
$$

and

$$
z=\left[h_{1}(0), h_{1}(1), \ldots, h_{1}\left(\frac{N_{1}}{2}-1\right)\right]^{T}
$$

where the superscript $T$ denotes the transpose operation. Using the above matrix notations, the overall design problem given by eqn. 13 can be reformulated as follows:

$$
\text { minimise }\left\|\mathrm{d}-\underline{\Omega}\left[\begin{array}{ll}
\mathbf{y}^{T} & \mathrm{z}^{T}
\end{array}\right]^{T}\right\|
$$

where

$$
\begin{aligned}
& \mathbf{d}=\left[\begin{array}{lc}
\mathbf{1}_{K+2}^{T} & \mathbf{0}_{K+2}^{T}
\end{array}\right]^{T} \\
& \Omega=\left[\begin{array}{cc}
\frac{1}{\sqrt{L L_{0}}} \mathbf{U}_{0 p} & \mathbf{O}_{I \times \frac{N_{1}}{2}} \\
\mathbf{O}_{(K-J+1) \times \frac{N_{0}}{2}} & \frac{1}{\sqrt{L L 1}} \mathbf{U}_{1 p} \\
\mathbf{U}_{a t} & \mathbf{U}_{b t} \\
\frac{r}{\sqrt{L L_{0}}} \mathbf{U}_{o s} & \mathbf{O}_{(K-J+1) \times \frac{N_{1}}{2}} \\
\mathbf{O}_{I \times \frac{N_{0}}{2}} & \frac{r}{\sqrt{L} \overline{L_{1}}} \mathbf{U}_{1 s} \\
\frac{r}{\sqrt{L} L_{1}} \mathbf{U}_{o t} & \frac{-r}{\sqrt{L} \mathbf{U}_{1}} \mathbf{U}_{1 t}
\end{array}\right]
\end{aligned}
$$

$\|x\|$ denotes the Chebyshev norm or peak of $x .0$ and $O$ represent a zero vector and a zero matrix with size shown by its subscript, respectively.

\subsubsection{Matrices used for formulation of the design problem:}

$\mathbf{U}_{0}=\left[u_{0}(i, j)\right]$, where

$$
\begin{aligned}
& u_{0}(i, j)=2 \cos \left\{\left(\frac{N_{0}+1}{2}-j\right) \omega_{i}\right\}, \\
& 1 \leq i \leq K, \quad 1 \leq j \leq \frac{N_{0}}{2}
\end{aligned}
$$

$\mathbf{U}_{1}=\left[u_{1}(i, j)\right]$, where

$$
\begin{aligned}
& u_{1}(i, j)=2 \sin \left\{\left(\frac{N_{1}+1}{2}-j\right) \omega_{i}\right\}, \\
& 1 \leq i \leq K, \quad 1 \leq j \leq \frac{N_{1}}{2}
\end{aligned}
$$

$\mathbf{U}_{0 p}=\left[u_{0 p}(i, j)\right]$, where

$$
\begin{aligned}
& u_{0 p}(i, j)=2 \cos \left\{\left(\frac{N_{0}+1}{2}-j\right) \omega_{i}\right\}, \\
& 1 \leq i \leq I, \quad 1 \leq j \leq \frac{N_{0}}{2}
\end{aligned}
$$

IEE Proc.-Vis. Image Signal Process., Vol. 145, No. 2, April 1998 
$\mathbf{U}_{1 p}=\left[u_{1 p}(i, j)\right]$, where

$$
\begin{aligned}
& u_{1 p}(i, j)=2 \sin \left\{\left(\frac{N_{1}+1}{2}-j\right) \omega_{i+J-1}\right\}, \\
& 1 \leq i \leq K-J+1, \quad 1 \leq j \leq \frac{N_{1}}{2}
\end{aligned}
$$

$\mathbf{U}_{0 s}=\left[u_{0 s}(i, j)\right]$, where

$$
\begin{aligned}
& u_{0 s}(i, j)=2 \cos \left\{\left(\frac{N_{0}+1}{2}-j\right) \omega_{i+J-1}\right\}, \\
& 1 \leq i \leq K-J+1, \quad 1 \leq j \leq \frac{N_{0}}{2}
\end{aligned}
$$

$\mathbf{U}_{1 s}=\left[u_{1 s}(i, j)\right]$, where

$$
\begin{aligned}
& u_{1 s}(i, j)=2 \sin \left\{\left(\frac{N_{1}+1}{2}-j\right) \omega_{i}\right\}, \\
& 1 \leq i \leq I, \quad 1 \leq j \leq \frac{N_{1}}{2}
\end{aligned}
$$

$\mathbf{U}_{0 t}=\left[u_{0 t}(i, j)\right]$, where

$$
\begin{aligned}
& u_{0 t}(i, j)=2 \cos \left\{\left(\frac{N_{0}+1}{2}-j\right) \omega_{i+I-1}\right\}, \\
& 1 \leq i \leq J-I+1, \quad 1 \leq j \leq \frac{N_{0}}{2}
\end{aligned}
$$

$\mathbf{U}_{1 t}=\left[u_{1 t}(i, j)\right]$, where

$u_{1 t}(i, j)=2 \sin \left\{\left(\frac{N_{1}+1}{2}-j\right)\left(\omega_{p}+\omega_{s}-\omega_{i+I-1}\right\}\right.$,

$1 \leq i \leq J-I+1, \quad 1 \leq j \leq \frac{N_{1}}{2}$

$\mathbf{U}_{a t}=\left[\mathrm{u}_{a t}(i, j)\right]$, where

$$
\begin{aligned}
u_{a t}(i, j)= & \frac{2 \tilde{H}_{0}\left(\omega_{i+I-1}\right)}{L L_{0} \sqrt{\tilde{T}\left(\omega_{i+I-1}\right)}} \\
& \times \cos \left\{\left(\frac{N_{0}+1}{2}-j\right) \omega_{i+I-1}\right\},
\end{aligned}
$$

$$
1 \leq i \leq J-I+1, \quad 1 \leq j \leq \frac{N_{0}}{2}
$$

$\mathbf{U}_{b t}=\left[\mathrm{u}_{b t}(i, j)\right]$, where

$$
\begin{aligned}
& u_{b t}(i, j)= \frac{2 \tilde{H}_{1}\left(\omega_{i+I-1}\right)}{L L_{1} \sqrt{\tilde{T}\left(\omega_{i+I-1}\right)}} \\
& \times \sin \left\{\left(\frac{N_{1}+1}{2}-j\right) \omega_{i+I-1}\right\}, \\
& 1 \leq i \leq J-I+1, \quad 1 \leq j \leq \frac{N_{1}}{2}
\end{aligned}
$$

\subsection{Proposed design method}

Based on the formulation given by eqn. 15 for designing the two-channel NDF bank with continuous coefficients, we present an efficient design method based on a modified dual-affine scaling (MDAS) variant of Karmarkar's algorithm to solve the considered design problem. The original and several modified versions of Karmarkar's algorithm have been successfully used for solving a variety of optimisation problems in the literature [9-12].

First, the design problem of eqn. 15 can be reformulated as follows:

$$
\begin{aligned}
& \text { minimise } \delta \\
& \text { subject to }\left\{\begin{array}{l}
\underline{\Omega} \mathbf{h}-\mathbf{d} \leq \Delta \\
-\underline{\Omega} \mathbf{h}+\mathbf{d} \leq \Delta
\end{array}\right.
\end{aligned}
$$

where $\Delta$ is a $(2 K+4) \times 1$ column vector with all entries equal to $\delta$ and $\mathbf{h}=\left[\mathbf{y}^{T} \mathbf{z}^{T}\right]^{T}$. Next, we further construct the following matrices:

$$
\begin{aligned}
& \mathbf{w}=\left[\begin{array}{ll}
\mathbf{h}^{T} & \delta
\end{array}\right]^{T}, \quad \mathbf{b}=\left[\begin{array}{ll}
\mathbf{0}^{T} & -1
\end{array}\right]^{T} \\
& \mathbf{c}=\left[\begin{array}{ll}
\mathbf{d}^{T} & -\mathbf{d}^{T}
\end{array}\right]^{T}, \quad \mathbf{A}=\left[\begin{array}{cc}
\frac{\Omega^{T}}{-\mathbf{1}^{T}} & -\mathbf{\Omega}^{T} \\
-\mathbf{1}^{T}
\end{array}\right]
\end{aligned}
$$

where $\mathbf{0}$ and $\mathbf{1}$ represent two vectors with appropriate sizes and all entries equal to zero and one, respectively. Accordingly, the minimax optimisation problem of eqn. 17 can be rewritten in the form of a dual optimisation problem as follows:

$$
\begin{aligned}
& \text { maximise } \mathbf{b}^{T} \mathbf{w} \\
& \text { subject to } \mathbf{A}^{T} \mathbf{w} \leq \mathbf{c}
\end{aligned}
$$

Based on the dual-affine scaling variant of Karmarkar's algorithm presented in [9], we introduce slack variables to the formulation of eqn. 19. This leads to the following optimisation problem which is equivalent to eqn. 19:

$$
\begin{aligned}
& \operatorname{maximise} \mathbf{b}^{T} \mathbf{w} \\
& \text { subject to } \mathbf{A}^{T} \mathbf{w}+\mathbf{v}=\mathbf{c}, \mathbf{v} \geq 0
\end{aligned}
$$

where $\mathbf{v}$ is the vector containing the slack variables.

Next, assume that we have an interior feasible solution $\mathbf{w}^{0}$ which satisfies $\mathbf{A}^{T} \mathbf{w}^{0}+\mathbf{v}^{0}=\mathbf{c}$ and $\mathbf{v}^{0}>0$ at the initial stage. With the initial solutions $\mathbf{w}^{0}$ and $\mathbf{v}^{0}$, it has been shown in [13] that an appropriate scaling operation must be performed to update $\mathbf{w}$ and $\mathbf{v}$ such that the objective function $\mathbf{b}^{T} \mathbf{w}$ can be improved at a faster rate. In [9], it was proposed to scale the slack variables as follows:

$$
\hat{\mathbf{v}}=\mathbf{D}_{v}^{-1} \mathbf{v}
$$

where

$$
\mathbf{D}_{v}=\operatorname{diag}(\mathbf{v})
$$

Substituting eqn. 21 into eqn. 20, we obtain

$$
\begin{aligned}
& \text { maximise } \mathbf{b}^{T} \mathbf{w} \\
& \text { subject to } \mathbf{A}^{T} \mathbf{w}+\mathbf{D}_{v} \hat{\mathbf{v}}=\mathbf{c}, \hat{\mathbf{v}} \geq 0
\end{aligned}
$$

Let the set of feasible solutions for eqn. 20 be given by

$$
\mathbf{W}=\left\{\mathbf{w} \in \mathbf{R}^{N_{n}+1} \mid \mathbf{A}^{T} \mathbf{w} \leq \mathbf{c}\right\}
$$

where $N_{n}=\left(N_{0}+N_{1}\right) / 2$. Then, the set of feasible scaled slack vectors for eqn. 23 is given by

$$
\hat{\mathbf{V}}=\left\{\hat{\mathbf{v}} \in \mathbf{R}^{4 K+8} \mid \exists \mathbf{w} \in \mathbf{W}, \mathbf{A}^{T} \mathbf{w}+\mathbf{D}_{v} \hat{\mathbf{v}}=\mathbf{c}\right\}
$$

From eqn. 25, it is easy to show that the corresponding $\mathbf{w}$ in $\mathbf{W}$, for a given scaled slack vector $\hat{\mathbf{v}}$ in $\hat{\mathbf{V}}$ is given by

$$
\mathbf{w}(\hat{\mathbf{v}})=\left(\mathbf{A} \mathbf{D}_{v}^{-2} \mathbf{A}^{T}\right)^{-1} \mathbf{A} \mathbf{D}_{v}^{-1}\left(\mathbf{D}_{v}^{-1} \mathbf{c}-\hat{\mathbf{v}}\right)
$$

and the one-to-one relationship between the feasible directions $\mathbf{f}_{w}$ in $\mathbf{W}$ and $\mathbf{f}_{\hat{v}}$ in $\hat{\mathbf{V}}$ is given by

$$
\mathbf{f}_{\hat{v}}=-\mathbf{D}_{v}^{-1} \mathbf{A}^{T} \mathbf{f}_{w}
$$

Based on eqns. 23 and 26, the feasible direction $\mathbf{f}_{\hat{\gamma}}$, can be obtained by computing the gradient of the objective function $\mathbf{b}^{T} \mathbf{W}$ with respect to $\hat{\mathbf{v}}$ as follows:

$$
\mathbf{f}_{\hat{v}}=\nabla_{\hat{v}}\left(\mathbf{b}^{T}(\mathbf{w}(\hat{\mathbf{v}}))\right)=-\mathbf{D}_{v}^{-1} \mathbf{A}^{T}\left(\mathbf{A} \mathbf{D}_{v}^{-2} \mathbf{A}^{T}\right)^{-1} \mathbf{b}
$$

Comparing eqns. 27 and 28 , we obtain

$$
\mathbf{f}_{w}=\left(\mathbf{A} \mathbf{D}_{v}^{-2} \mathbf{A}^{T}\right)^{-1} \mathbf{b}
$$


After determining $\mathbf{f}_{w}$ from eqn. 29 , we note that updating $\mathbf{w}$ can be carried out as follows:

$$
\mathbf{w}^{i+1}=\mathbf{w}^{i}+\alpha \mathbf{f}_{w}=\left[\begin{array}{l}
\mathbf{h}^{i} \\
\delta^{i}
\end{array}\right]+\alpha\left[\begin{array}{l}
\mathbf{f}_{h} \\
f_{\delta}
\end{array}\right]
$$

if a suitable step size $\alpha$ is found, where $\mathbf{w}^{i}, \mathbf{h}^{i}$, and $\delta$ represent the $\mathbf{w}, \mathbf{h}$, and $\delta$ obtained after the $(i-1)$ th iteration, respectively, during the optimisation process. We use this $\mathbf{f}_{h}$, which is the subvector containing the first $N_{n}$ entries of the feasible direction $\mathbf{f}_{w}$, as the true descent direction for updating the coefficient vector $\mathbf{h}$ of the optimisation problem in eqn. 23. To find a suitable step size $\alpha$ analytically, instead of numerically, for updating $\mathbf{w}$, we propose an efficient method by considering the feasibility of using the updated slack variable $\mathbf{v}^{i}+\alpha \mathbf{f}_{w}$. First, from eqns. 21 and 28 , we have the feasible direction for the unscaled slack variable as follows:

$$
\mathbf{f}_{v}=-\mathbf{A}^{T}\left(\mathbf{A} \mathbf{D}_{v}^{-2} \mathbf{A}^{T}\right)^{-1} \mathbf{b}=-\mathbf{A}^{T} \mathbf{f}_{w}
$$

Then, based on the fact that the updated slack vector $\mathbf{v}$ must be a vector with all entries greater than or equal to zero, a suitable step size $\alpha$ can be obtained by taking the most appropriate feasible step in the direction of $\mathbf{f}_{v}$ as follows:

$$
\alpha=\gamma \times \min \left\{-\frac{\left(\mathbf{v}^{i}\right)_{j}}{\left(\mathbf{f}_{v}\right)_{j}} \mid\left(\mathbf{f}_{v}\right)_{j}<0\right\}
$$

where $0<\gamma<1$ is in general chosen experimentally. $(\mathbf{z})_{j}$ denotes the $j$ th entry of the vector $\mathbf{z}$ and $\min \{x\}$ the minimum value of $x$. Based on eqns. 18 ans 30 , the formula for updating $\mathbf{h}$ after the $i$ th iteration is then given by

$$
\mathbf{h}^{i+1}=\mathbf{h}^{i}+\alpha \mathbf{f}_{h}
$$

\subsection{Selection of the Rrequired initial guess}

Based on the proposed design method presented in Section 3.2, the overall design process is basically an iterative procedure. To initiate the iteration, an initial guess for the filter coefficient vector $\mathbf{h}$ must be provided. As the initial guess will affect the convergence speed and the design results, an appropriate initial guess is usually the one which produces the best design results in several iterations. For the considered design problem, our design experience shows that an appropriate initial guess $\mathbf{h}^{0}=\left[\mathbf{y}^{0} \mathbf{z}^{0}\right)$ of $\mathbf{h}$ can be obtained as follows:

First, we compute the unconstrained least-squares solutions given by

$$
\begin{aligned}
& \mathbf{y}^{0}=\sqrt{L L_{0}}\left(\mathbf{U}_{0 p}^{T} \mathbf{U}_{0 p}+\mathbf{U}_{0 s}^{T} \mathbf{U}_{0 s}\right)^{-1}\left(\mathbf{U}_{0 p}^{T} \mathbf{1}_{I}\right) \\
& \mathbf{z}^{0}=\sqrt{L L_{1}}\left(\mathbf{U}_{1 p}^{T} \mathbf{U}_{1 p}+\mathbf{U}_{1 s}^{T} \mathbf{U}_{1 s}\right)^{-1}\left(\mathbf{U}_{1 p}^{T} \mathbf{1}_{K-J+1}\right)
\end{aligned}
$$

and compute the magnitude responses $\hat{H}_{0}(\omega)$ of $H_{0}(z)$, $\hat{H}_{1}(\omega)$ of $H_{1}(z)$ and the magnitude response $T(\omega)$ of the NDF bank corresponding to $\mathbf{h}^{0}$, respectively. Next, utilising the WLS algorithm presented by one of the authors in [14] to obtain a weighted least-squares solution as follows: Construct the required envelop function $B(\omega)$ from the error function $|\hat{T}(\omega)-1|$ according to the WLS algorithm of [14]. Based on $B(\omega)$, we compute the required update function $v(\omega)$ according to the following formula:

$$
\nu(\omega)=\frac{(2 K+4)\{B(\omega)\}^{1.5}}{\sum_{k=1}^{(2 K+4)}\left[\hat{W}\left(\omega_{k}\right)\left\{B\left(\omega_{k}\right)\right\}^{1.5}\right]}
$$

where $\hat{W}(\omega)$ denotes the initial weighting function which is set to one for all $\omega$. Then, update the weighting function as $W(\omega)=\hat{W}(\omega) v(\omega)$ and form the associated weighting matrix $\mathbf{W}=\operatorname{diag}\left(W\left(\omega_{1}\right), W\left(\omega_{2}\right), \ldots\right.$, $W\left(\omega_{K}\right)$ ), where $\operatorname{diag}()$ denotes a diagonal matrix.

Finally, we solve the following linear equations to find the appropriate initial filter coefficient vector $\mathbf{h}^{0}$ :

$$
\left[\begin{array}{cc}
\hat{\mathbf{A}} & \hat{\mathbf{B}} \\
\hat{\mathbf{B}}^{T} & \hat{\mathbf{A}}
\end{array}\right]\left[\begin{array}{l}
\mathbf{y}^{0} \\
\mathbf{z}^{0}
\end{array}\right]=\left[\begin{array}{l}
\mathbf{U}_{a}^{T} \mathbf{W} 1_{K} \\
\mathbf{U}_{b}^{T} \mathbf{W} \mathbf{1}_{K}
\end{array}\right]
$$

where $\hat{\mathbf{A}}=\mathbf{U}_{a}^{T} \mathbf{W} \mathbf{U}_{a}+\mathbf{U}_{0 s}^{T} \mathbf{U}_{0 s}+\mathbf{U}_{0 t}^{T} \mathbf{U}_{0 t}, \hat{\mathbf{B}}=\mathbf{U}_{a}^{T} \mathbf{W} \mathbf{U}_{b}-$ $\mathbf{U}_{0 t}^{T} \mathbf{U}_{1 t}, \hat{\mathbf{C}}=\mathbf{U}_{b}^{T} \mathbf{W} \mathbf{U}_{b}+\mathbf{U}_{1 s}^{T} \mathbf{U}_{1 s}+\mathbf{U}_{1 t}^{T} \mathbf{U}_{1 t}, \mathbf{U}_{a}=\mathbf{H}_{0} \mathbf{U}_{0}$, $\mathbf{U}_{b}=\mathbf{H}_{1} \mathbf{U}_{1}, \quad \mathbf{H}_{0}=1 / L L_{0} \operatorname{diag}\left(\hat{H}_{0}\left(\omega_{1}\right), \quad \hat{H}_{0}\left(\omega_{2}\right), \ldots\right.$, $\left.\hat{H}_{0}\left(\omega_{K}\right)\right)$ and $\mathbf{H}_{1}=1 / L L_{0} \operatorname{diag}\left(\hat{H}_{1}\left(\omega_{1}\right), \quad \hat{H}_{1}\left(\omega_{2}\right), \ldots\right.$, $\left.\hat{H}_{1}\left(\omega_{K}\right)\right)$. Using the initial filter coefficients obtained from eqn. 35 , we then compute the corresponding magnitude responses $H_{0}^{0}(\omega)$ and $H_{1}^{0}(\omega)$. Moreover, we use the $H_{0}^{0}(\omega)$ and $H_{1}^{0}(\omega)$ as the initial guesses of $\tilde{H}_{0}(\omega)$ and $\tilde{H}_{1}(\omega)$, respectively.

\subsection{The design procedure}

From the results in Section 3.3, we summarise the proposed design technique by presenting the following design procedure:

Step 1: Initialise the design process. Set the iteration number $i=0$.

1.1 Specify the design parameters: the filter lengths $N_{0}$ and $N_{1}$ the bandedge frequencies $\omega_{p}$ and $\omega_{s}$ and the ripple ratio $r$. Construct the matrices $\mathbf{b}$ and $\mathbf{c}$ from eqn. 18.

1.2 Use the method presented in Section 3.3 to generate a suitable initial guess $\mathbf{h}^{0}$ for the coefficient vector $\mathbf{h}$ and construct the matrix $\Omega$ corresponding to $\mathbf{h}^{0}$.

1.3 Compute corresponding initial magnitude responses $H_{0}^{0}(\omega)$ and $H_{1}^{0}(\omega)$, and set $H_{0}^{0}(\omega)$ and $H_{1}^{0}(\omega)$ as the initial guesses for $\tilde{H}_{0}(\omega)$ and $\tilde{H}_{1}(\omega)$, respectively. Form the initial parameter vector

$$
\mathbf{w}^{0}=\left[\begin{array}{c}
\mathbf{h}^{0} \\
\delta^{0}
\end{array}\right]
$$

where $\delta^{0}=1.01 \max \left\{\left|\mathbf{d}-\Omega \mathbf{h}^{0}\right|\right\}$.

Step 2; At the ith iteration, calculate the matrices $\Omega$ and A corresponding to the current filter coefficient vector $\mathbf{h}^{i}$. Then, calculate the corresponding error vector $\mathbf{e}^{i}=\Omega \mathbf{h}^{i}-\mathbf{d}$ and the peak ripple $\delta^{i}=1.01$ $\max \left\{\left|\mathbf{e}^{i}\right|\right\}$.

Step 3: Find the slack vector $\mathbf{v}^{i}=\mathbf{c}-\mathbf{A}^{T} \mathbf{w}^{i}=\left[\begin{array}{ll}\mathbf{v}_{1}^{T} & \mathbf{v}_{2}^{T}\end{array}\right]^{T}$, where $\mathbf{v}_{1}=\delta^{i} \mathbf{1}-\mathbf{e}^{i}, \mathbf{v}_{2}=\delta^{i} \mathbf{1}+\mathbf{e}^{i}$.

Step 4: Compute the feasible direction vector $\mathbf{f}_{w}=$ $\left(\mathbf{A D}_{v}^{-2} \mathbf{A}^{T}\right)^{-1} \mathbf{b}$ for optimisation. Instead of directly computing $\mathbf{f}_{w}$, by performing the inverse of $\left(\mathbf{A D}_{v}^{-2} \mathbf{A}^{T}\right)$, we propose an efficient algorithm as follows:

4.1 Construct the diagonal matrices $\mathbf{D}_{1}=\operatorname{diag}\left(\mathbf{v}_{1}\right)$, $\mathbf{D}_{2}=\operatorname{diag}\left(\mathbf{v}_{2}\right)$, and $\mathbf{D}=\left(\mathbf{D}_{1}^{-2}+\mathbf{D}_{2}^{-2}\right)$.

4.2 Compute the column vector $\mathbf{x}_{1}=\Omega^{T}\left(\mathbf{D}_{2}^{-2}-\mathbf{D}_{1}^{-2}\right) \mathbf{1}$ and the value $c_{1}=-\left(\mathbf{1}^{T} \mathbf{D} \mathbf{1}\right)^{-1}$.

4.3 Solve the equation $\left(\Omega^{T} \mathbf{D} \Omega+c_{1} \mathbf{x}_{1} \mathbf{x}_{1}{ }^{T}\right) \mathbf{f}_{h}=-c_{1} \mathbf{x}_{1}$ using Gaussian elimination to find $\mathbf{f}_{h}$.

4.4 Compute $f_{\delta}=c_{1}\left(\mathbf{x}_{1}{ }^{T} \mathbf{f}_{h}+1\right)$. Then, form the desired direction vector $\mathbf{f}_{w}=\left[\mathbf{f}_{h}^{T} f_{\delta}\right]^{T}$.

Step 5: Compute the feasible direction vector $\mathbf{f}_{v}=$ $-\mathbf{A}^{T} \mathbf{f}_{w}=\left[\mathbf{f}_{v 1}^{T} \mathbf{f}_{v 2}^{T}\right]^{T}$, where $\mathbf{f}_{v 1}=\mathbf{f}_{\delta} \mathbf{1}-\boldsymbol{\Omega} \mathbf{f}_{h}, \mathbf{f}_{v 2}=f_{\delta} \mathbf{1}+\boldsymbol{\Omega} \mathbf{f}_{h}$. Step 6: Determine the step size $\alpha$ according to eqn. 32 . 
Then, update the filter coefficient vector $\mathbf{h}$ according to eqn. 33 and calculate the associated peak error $\delta^{i+1}=\delta^{i}$ $+\alpha f_{\delta}$ according to eqn. 30 .

Step 7: Define a performance indication function

$$
\epsilon(i+1)=\left|\frac{\delta^{i}-\delta^{i+1}}{\delta^{i}}\right|
$$

It is reasonable to terminate the design process whenever the indication function is small enough. Therefore, we stop the iterative procedure if $\varepsilon(i+1) \leq \kappa$, where $\kappa$ is a preset small positive real number. Otherwise, set $\tilde{H}_{0}(\omega)=H_{0}^{i+1}(\omega), \tilde{H}_{1}(\omega)=H_{1}^{i+1}(\omega)$, and $i=i+1$, then go to Step 2.

\section{Simulation examples}

In this Section, we present several simulation examples of designing two-channel NDF banks with linear-phase FIR filters for illustration. These designs are performed on a personal computer with Pentium CPU using MATLAB programming language. For all design examples, the number $K$ of frequency grid points used is set to $8 \times \max \left(N_{0}, N_{1}\right)$. Moreover, the ripple ratio $r$ for the design problem shown by eqn. 13 is set to 0.25 . The value of the $\gamma$ required by eqn. 32 is set to 0.99 . The value of the $\kappa$ used for terminating the design process is $10^{-6}$. The performance for each of the designed filter banks is evaluated in terms of the peak reconstruction error (PRE) in $\mathrm{dB}$, the normalised peak passband ripple (NPPR) in $\mathrm{dB}$, the normalised peak stopband ripple (NPSR) in $\mathrm{dB}$, and the stopband ripple energies (SRE) of the designed $H_{0}(z)$ and $H_{1}(z)$. They are defined as follows:

$$
\begin{array}{ll}
\mathrm{PRE}=\max \left\{\left|20 \log _{10} T(\omega)\right|\right\} \quad \text { for } w \in[0, \pi] \\
\mathrm{NPSR}_{0}=\max \left\{20 \log _{10} \frac{\left|H_{0}(\omega)\right|}{\left.\sqrt{L L_{0}}\right\}} \text { for } \omega \in\left[\omega_{s}, \pi\right]\right. \\
\mathrm{NPSR}_{1}=\max \left\{20 \log _{10} \frac{\left|H_{1}(\omega)\right|}{\left.\sqrt{L L_{1}}\right\}} \text { for } \omega \in\left[0, \omega_{p}\right]\right. \\
\mathrm{SRE}_{0}=\int_{\omega_{s}}^{\pi} H_{0}^{2}(w) d \omega \text { and } \mathrm{SRE}_{1}=\int_{0}^{\omega_{p}} H_{1}^{2}(\omega) d \omega
\end{array}
$$

Example 1: The design specifications used are shown by case 1 in Table 1 . Table 2 lists the significant design results after 25 iterations. The design results obtained from [8], based on the least-absolute error criterion, are also shown for comparison. We observe that the proposed technique produces much smaller peak ripples than [8]. Table 3 shows the filter coefficients of the designed analysis filters $H_{0}(z)$ and $H_{1}(z)$ for this case. Fig. 3 plots the corresponding magnitude responses in $\mathrm{dB}$, of $H_{0}(\omega) / \sqrt{ }\left(L L_{0}\right)$ and $H_{1}(\omega) / \sqrt{ }\left(L L_{1}\right)$, and the overall magnitude response $T(\omega)$ in $\mathrm{dB}$ of the designed NDF bank. We note that the designed NDF bank shows satisfactory performance.

Table 1: Design specifications for examples 1 and 2

\begin{tabular}{lllllll}
\hline & $N_{0}$ & $N_{1}$ & $\omega_{p}$ & $\omega_{s}$ & $L_{0}$ & $L_{1}$ \\
\hline Case 1 & 32 & 32 & $0.3 \pi$ & $0.5 \pi$ & 2 & 3 \\
Case 2 & 80 & 80 & $0.16 \pi$ & $0.24 \pi$ & 1 & 4 \\
\hline
\end{tabular}

Table 2: Significant design results for examples 1 and 2

\begin{tabular}{lll}
\hline Example 1 & Proposed method & Method of [7] \\
\hline PRE, dB & 0.03161 & 0.04377 \\
NPSR $(\mathrm{dB})$ of $H_{0}(\omega)$ & -42.8854 & -29.7787 \\
NPSR $(\mathrm{dB})$ of $H_{1}(\omega)$ & -42.9158 & -34.0271 \\
SRE of $H_{0}(\omega)$ & $4.0432 \times 10^{-4}$ & $1.5554 \times 10^{-4}$ \\
SRE of $H_{1}(\omega)$ & $3.6990 \times 10^{-4}$ & $1.4742 \times 10^{-4}$ \\
\hline Example 2 & Proposed method & Method of [7] \\
\hline PRE, dB & 0.0254 & 0.0206 \\
NPSR $(\mathrm{dB})$ of $H_{0}(\omega)$ & -43.7402 & -26.5533 \\
NPSR $(\mathrm{dB})$ of $H_{1}(\omega)$ & -45.0486 & -32.3882 \\
SRE of $H_{0}(\omega)$ & $1.8594 \times 10^{-4}$ & $4.8525 \times 10^{-5}$ \\
SRE of $H_{1}(\omega)$ & $1.6150 \times 10^{-4}$ & $2.2802 \times 10^{-4}$ \\
\hline
\end{tabular}
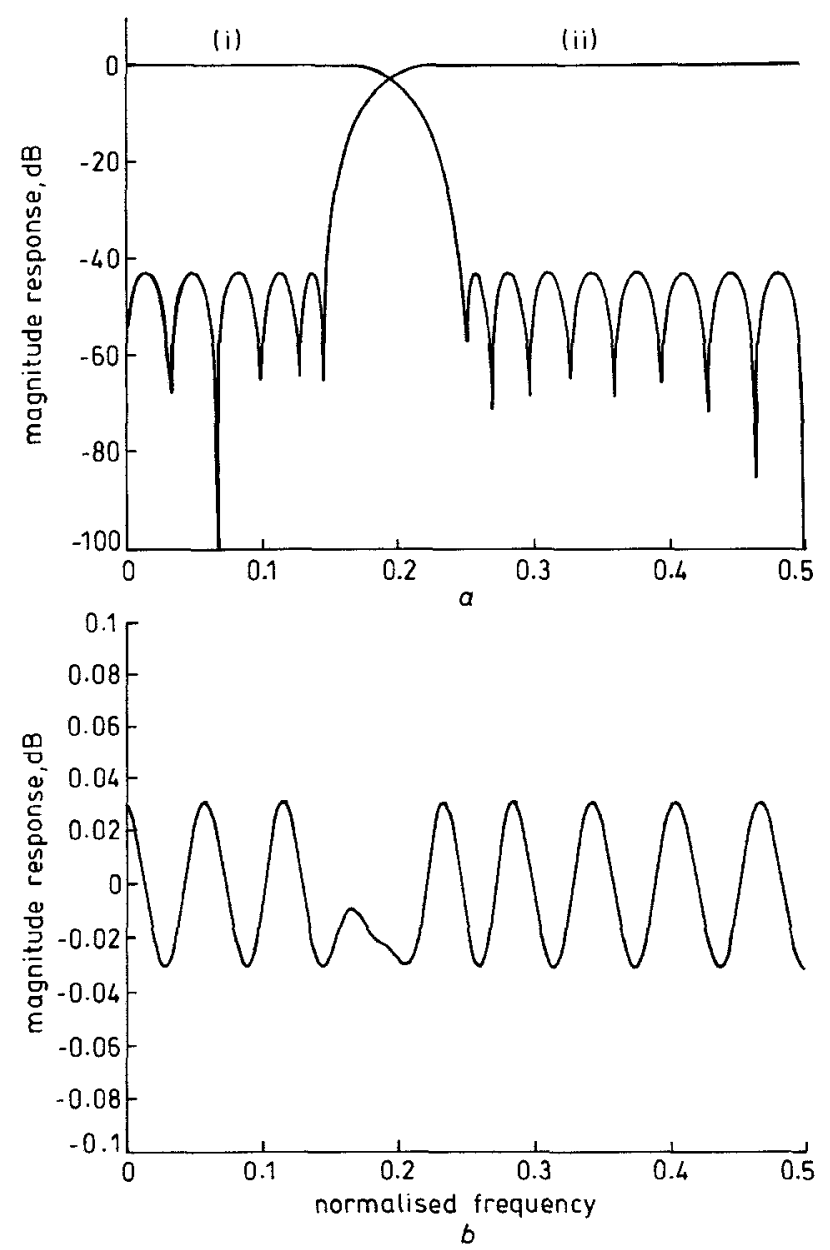

Fig.3 Results for example 1

a Magnitude responses of the designed analysis filters; (i) $H_{0}(\omega) / \sqrt{ }\left(L L_{0}\right)$; (ii) $H_{1}(\omega) / \sqrt{ }\left(L L_{1}\right)$

$b$ Magnitude response of the designed filter bank

Example 2: The design specifications used are shown by case 2 in Table 1 . Table 2 lists the significant design results after 24 iterations. The design results obtained from [8], based on the least-absolute error criterion, are also shown for comparison. We note that the proposed technique produces much smaller NPSR for $H_{0}(\omega)$ and $H_{1}(\omega)$ than [8], although the obtained PRE is about $0.005 \mathrm{~dB}$ larger than [8]. The filter coefficients of the designed analysis filters $H_{0}(z)$ and $H_{1}(z)$, for this case, are shown in Table 4. Fig. 4 depicts the corresponding magnitude responses, in $\mathrm{dB}$, of $H_{0}(\omega) / \sqrt{ }\left(L L_{0}\right)$ and $H_{1}(\omega) / \sqrt{ }\left(L L_{1}\right)$ and the overall magnitude response $T(\omega)$, in $\mathrm{dB}$, of the designed NDF bank. Again, we observe 
that the designed NDF bank shows satisfactory performance.

Table 3: Coefficients of the designed filters $H_{0}(z)$ and $H_{1}(z)$ for example 1

\begin{tabular}{rrrl}
\hline$n$ & \multicolumn{1}{l}{$h_{0}(n)$} & \multicolumn{1}{l}{$h_{1}(n)$} & $n$ \\
\hline 0 & 1.23618061095655 & 2.03878258718131 & 31 \\
1 & 0.60366368814555 & -0.17085591494049 & 30 \\
2 & -0.06593423400779 & -0.46462363986519 & 29 \\
3 & -0.26250252196451 & -0.16895594321699 & 28 \\
4 & -0.05978007203571 & 0.14027327860362 & 27 \\
5 & 0.12458210425695 & 0.17385811080191 & 26 \\
6 & 0.08099324386912 & 0.01871376513089 & 25 \\
7 & -0.04535651182571 & -0.09339193740865 & 24 \\
8 & -0.06563937131828 & -0.06633719998891 & 23 \\
9 & 0.00403015855316 & 0.01689334155360 & 22 \\
10 & 0.04055005846851 & 0.04892162177594 & 21 \\
11 & 0.01116042939344 & 0.01852213686230 & 20 \\
12 & -0.01873820489688 & -0.01720549184744 & 19 \\
13 & -0.01445539655885 & -0.02146315346314 & 18 \\
14 & 0.01185217520092 & -0.00683499670667 & 17 \\
15 & 0.00335802636641 & 0.00405233023957 & 16 \\
\hline
\end{tabular}
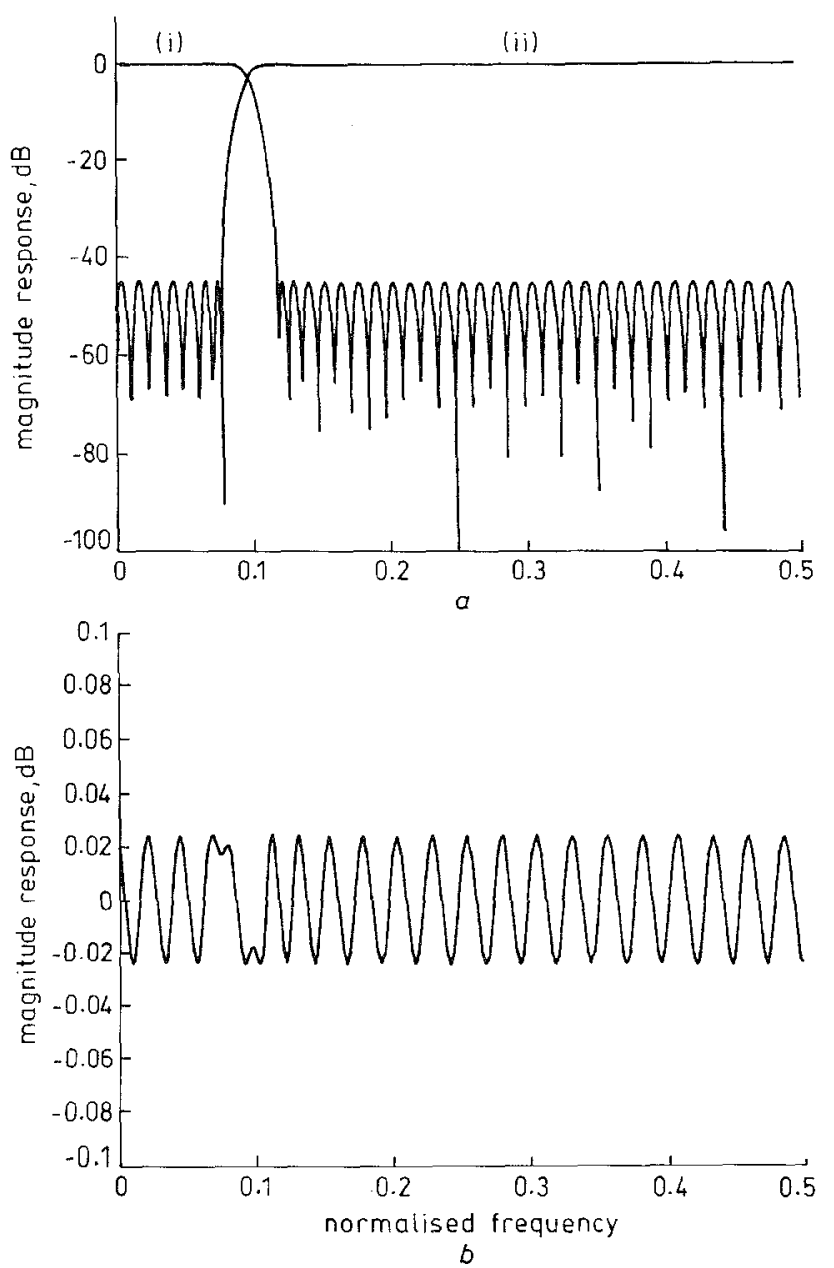

Fig.4 Results for example 2

a Magnitude responses of the designed analysis filters; (i) $H_{0}(\omega) / V\left(L L_{0}\right)$; (ii) $H_{1}(\omega) / V\left(L L_{1}\right)$

$b$ Magnitude response of the designed filter bank

\section{Conclusion}

This paper has presented a technique for the minimax design of two-channel nonuniform-division filter
Table 4: Coefficients of the designed filters $H_{0}(z)$ and $H_{1}(z)$ for example 2

\begin{tabular}{cccl}
\hline$n$ & $h_{0}(n)$ & \multicolumn{1}{l}{$h_{1}(n)$} & $n$ \\
\hline 0 & 0.45803124267317 & 2.71841395946488 & 79 \\
1 & 0.39391839289863 & 0.58653979383744 & 78 \\
2 & 0.28212798902572 & 0.03760272343259 & 77 \\
3 & 0.15045999110334 & -0.20458764522497 & 76 \\
4 & 0.02957640277630 & -0.28059364907837 & 75 \\
5 & -0.05593866670522 & -0.24672705482211 & 74 \\
6 & -0.09405098288370 & -0.15012893322837 & 73 \\
7 & -0.08738564264302 & -0.03549196098245 & 72 \\
8 & -0.05051530933699 & 0.06025689577293 & 71 \\
9 & -0.00378564696091 & 0.11407619720969 & 70 \\
10 & 0.03389393829676 & 0.11969115334170 & 69 \\
11 & 0.05083166321064 & 0.08597160778625 & 68 \\
12 & 0.04509235882373 & 0.03156805275825 & 67 \\
13 & 0.02331411153929 & -0.02232291227351 & 66 \\
14 & -0.00324964308123 & -0.05875000957058 & 65 \\
15 & -0.02365064588068 & -0.06940440350199 & 64 \\
16 & -0.03123670574243 & -0.05563974615065 & 63 \\
17 & -0.02540595923248 & -0.02633796252971 & 62 \\
18 & -0.0107945693549 & 0.00623448358185 & 61 \\
19 & 0.00539069612345 & 0.03094123405048 & 60 \\
20 & 0.01659802796562 & 0.04118237891154 & 59 \\
21 & 0.01934514515559 & 0.03623637168863 & 58 \\
22 & 0.01404992006621 & 0.02046137903336 & 57 \\
23 & 0.00423858802056 & 0.00096504172300 & 56 \\
24 & -0.00543803955506 & -0.01522284800301 & 55 \\
25 & -0.01117196006325 & -0.02345510603403 & 54 \\
26 & -0.01142137968840 & -0.02256204344689 & 53 \\
27 & -0.00715516359514 & -0.01460076673778 & 52 \\
28 & -0.00082908929037 & -0.00354834307683 & 51 \\
29 & 0.00443393816193 & 0.00638861799696 & 50 \\
30 & 0.00709035681695 & 0.01221907841134 & 49 \\
31 & 0.00600460723563 & 0.01291636622822 & 48 \\
32 & 0.00334026241524 & 0.00934740945378 & 47 \\
33 & -0.00099161144139 & 0.00357636799262 & 46 \\
34 & -0.00254982805010 & -0.00208799277315 & 45 \\
35 & -0.00494400133026 & -0.00593141524694 & 44 \\
36 & -0.00157439853624 & -0.00725715196422 & 43 \\
38 & -0.00372373281058 & -0.00634679156296 & 42 \\
39 & 0.00344441728518 & -0.00593579710025 & 41 \\
& 0.00422786323294 & -0.00009662845410 & 40 \\
\hline
\end{tabular}

(NDF) banks with linear-phase FIR filters. The design problem has been formulated as an optimisation problem based on a linearisation scheme. A design method has been developed based on the use of a modified dual-affine scaling variant of Karmarkar's algorithm for updating the filter coefficient vector. Appropriate selection of the initial guess has been presented for initiating the design process. Moreover, an analytical formula has been proposed for calculating the step size required at each iteration. As a result, the coefficients of the analysis filters can be obtained efficiently by solving only linear equations during the design process. Simulation results have shown the effectiveness of the proposed design technique. 


\section{Acknowledgment}

This work was supported by the National Science Council under Grant NSC86-2221-E002-025.

\section{References}

1 CROCHIERE, R.E.: 'Digital signal processor: sub-band coding', Bell Syst. Tech. J., 1981, 60, pp. 1633-1653

2 BELLANGER, M.G., and DAGUET, J.L.: 'TDM-FDM transmultiplexer: digital polyphase and FFT', IEEE Trans., 1974, COM-22, pp. 1199-1204

3 VARY, $P$., and HEUTE, $U$.: "A short-time spectrum analyzer with polyphase network and DFT', Signal Process., 1980, 2, pp. $55-65$

4 WOODS JW and O'NEIL S.D. 'Subband coding of images', IEEE Trans. Acoust. Speech Signal Process., 1986, ASSP-34, pp. $1278-1288$

5 KOVAC̈EVIĆ, J.: 'Filter banks and wavelets: Extensions and applications'. PhD . dissertation, Columbia University, New York, NY, October 1991

6 NAYEBI, K., BARNWELL, T.P., and SMITH, M.J.T.: 'Nonuniform filter banks: a reconstruction and design theory', IEEE Trans. Signal Process., 1993, 41, pp. 1114-1127

7 WADA, S: 'Design of nonuniform division multirate FIR filter banks', IEEE Trans. Circuits Syst. II. Analog Digit. Signal Process., 1995, 42, pp. 115-121

8 LEE, J.-H., and HUANG, S.-C.: 'Design of two-channel nonuniform-division maximally decimated filter banks using $\mathrm{L}_{1}$ criteria', IEE Proc. Vis. Image Signal Process., 1996, 143, (2), pp. 79-83

9 ADLER, I., KARMARKAR, N., RESENDE, M.G.C., and VEIGA, G.: 'An implementation of Karmarkar's algorithm for linear programming', Math. Program., 1989, 44, pp. 297-335

10 KARMARKAR, N.: 'A new polynomial-time algorithm for linear programming', Combinatorica, 1984, 4, pp. 373-395

11 VANDERBEI, R.J., MEKETON, M.S., and FREEDMAN, B.A.: 'A modification of Karmarkar's linear programming algorithm', Algorithmica, 1986,1 pp. 395-407

12 RUZINSKY, S.A., and OLSEN, E.T.: ' $\mathrm{L}_{1}$ and $\mathrm{L}_{\infty}$ minimization via a variant of Karmarkar's algorithm', IEEE Trans. Acoust. Speech Signal Process., 1989, ASSP-37, pp. 245-253

13 HILLER, F.S., and LIEBERMAN, G.J.: 'Introduction to mathematical programming' (McGraw-Hill, New York, 1991)

14 LIM, Y.-C., LEE, J.-H., CHEN, C.-K., and YANG, R.H.: 'A weighted least-squares algorithm for quasi-equiripple FIR and IIR digital filter design', IEEE Trans. Signal Process., 1992, 40, pp. $551-558$

15 RABINER, L.R., and GOLD, B.: 'Theory and application of digital signal processing' (Prentice-Hall, Englewood Cliffs, NJ, 1975)

\section{Appendix}

Let $B_{0}(z)$ and $B_{1}(z)$ be L.P FIR filters with lengths equal to $N_{b 0}$ and $N_{b 1}$, respectively. The associated maguitude responses are set to $B_{0}(\omega)=1$, for $\omega \in\left[0, \omega_{s} / L_{0}\right]$ and $=$ 0 , for $\omega \in\left[\left(2 \pi-\omega_{s}\right) / L_{0}, \pi\right]$ and $B_{1}(\omega)=1$, for $\omega \in[0$, $\left.\left(\pi-\omega_{p}\right) / L_{1}\right]$ and $=0$, for $\omega \in\left[\left(\pi+\omega_{p}\right) / L_{1}, \pi\right]$, respectively. Moreover, assume that $H_{0}(z)$ and $H_{1}(z)$ have zero stopband response. Then, it follows that the input/ output relationship of the NDF bank in the frequency domain is given by [8]

$$
\begin{aligned}
\hat{X}\left(e^{j \omega}\right)=\frac{e^{-j \omega G_{0}}}{L L_{0}}\left[X\left(e^{j \omega}\right) H_{0}\left(e^{j \omega}\right)\right. & \\
& +X\left(e^{j \omega} W_{L}^{L_{0}}\right) H_{0}\left(e^{j \omega} W_{L}^{L_{0}}\right) \\
& \left.+X\left(e^{j \omega} W_{L}^{-L_{0}}\right) H_{0}\left(e^{j \omega} W_{L}^{-L_{0}}\right)\right] F_{0}\left(e^{j \omega}\right) \\
+ & \frac{e^{-j \omega G_{1}}}{L L_{1}}\left[X\left(e^{j \omega}\right) H_{1}\left(e^{j \omega}\right)\right. \\
& +X\left(e^{j \omega} W_{L}^{L_{1}}\right) H_{1}\left(e^{j \omega} W_{L}^{L_{1}}\right) \\
& \left.+X\left(e^{j \omega} W_{L}^{-L_{1}}\right) H_{1}\left(e^{j \omega} W_{L}^{-L_{1}}\right)\right] F_{1}\left(e^{j \omega}\right)
\end{aligned}
$$

where $G_{0}=\left(N_{b 0}-1\right) / L_{0}$ and $G_{1}=\left(N_{b 1}-1\right) / L_{1}$ are set to integers to avoid noninteger group delays. The twiddle factors $W_{L}=\exp (-j 2 \pi / L)$. Substituting the conditions $L=L_{0}+L_{1}, F_{0}\left(e^{j \omega}\right)=H_{0}\left(e^{j \omega}\right)$ and $F_{1}\left(e^{j \omega}\right)=$
$-H_{1}\left(e^{j \omega}\right)$ into eqn. 37 yields

$$
\begin{aligned}
\hat{X}\left(e^{j \omega}\right)= & T\left(e^{j \omega}\right) X\left(e^{j \omega}\right)+A_{1}\left(e^{j \omega}\right) X\left(e^{j \omega} W_{L}^{L_{0}}\right) \\
& +A_{2}\left(e^{j \omega}\right) X\left(e^{j \omega} W_{L}^{L_{1}}\right)
\end{aligned}
$$

where

$$
\begin{aligned}
T\left(e^{j \omega}\right)= & \frac{e^{-j \omega G_{0}}}{L L_{0}} H_{0}^{2}\left(e^{j \omega}\right)-\frac{e^{-j \omega G_{1}}}{L L_{1}} H_{1}^{2}\left(e^{j \omega}\right) \\
A_{1}\left(e^{j \omega}\right)= & \frac{e^{-j \omega G_{0}}}{L L_{0}} H_{0}\left(e^{j \omega}\right) H_{0}\left(e^{j \omega} W_{L}^{L_{0}}\right) \\
& -\frac{e^{-j \omega G_{1}}}{L L_{1}} H_{1}\left(e^{j \omega}\right) H_{1}\left(e^{j \omega} W_{L}^{L_{0}}\right) \\
A_{2}\left(e^{j \omega}\right)= & \frac{e^{-j \omega G_{0}}}{L L_{0}} H_{0}\left(e^{j \omega}\right) H_{0}\left(e^{j \omega} W_{L}^{L_{1}}\right) \\
& -\frac{e^{-j \omega G_{1}}}{L L_{1}} H_{1}\left(e^{j \omega}\right) H_{1}\left(e^{j \omega} W_{L}^{L_{1}}\right)
\end{aligned}
$$

The first term of eqn. 38 represents the response of a linear shift-invariant system $T\left(e^{j \omega}\right)$ with input $X\left(e^{j \omega}\right)$, while the other two terms represent the resulting aliasing distortion. Therefore, perfect reconstruction requires the following conditions:

PR 1: The magnitude $T(\omega)$ of $T\left(e^{j \omega}\right)$ must be equal to 1, i.e. $T(\omega)=1$, for all $\omega$.

PR 2: The magnitude $A_{1}(\omega)$ of $A_{1}\left(e^{j \omega}\right)$ must be zero, i.e. $A_{1}(\omega)=0$, for all $\omega$.

PR 3: The magnitude $A_{2}(\omega)$ of $A_{2}\left(e^{j \omega}\right)$ must be zero, i.e. $A_{2}(\omega)=0$, for all $\omega$.

We note from eqn. 39 that $H_{0}(z)$ must be either a case 1 or case 2 LP FIR filter, while $H_{1}(z)$ must be a case 4 LP FIR filter to ensure the PR 1 condition. The definitions for each of the case 1 to 4 of LP FIR filters can be found in [15]. Let $H_{0}(z)$ and $H_{1}(z)$ be LP FIR filters with lengths equal to $N_{0}$ and $N_{1}$, respectively. Then, $H_{0}\left(e^{j \omega}\right)$ can be expressed as [15]

$$
H_{0}\left(e^{j \omega}\right)=e^{-j \frac{\left(N_{0}-1\right) \omega}{2}} H_{0}(\omega)
$$

where

$$
H_{0}(\omega)= \begin{cases}h_{0}\left(\frac{N_{0}-1}{2}\right)+\sum_{n=0}^{\frac{N_{0}-3}{2}} 2 h_{0}(n) \cos \left(\omega\left(n-\frac{N_{0}-1}{2}\right)\right) \\ \text { for case } 1 \\ \sum_{n=0}^{\frac{N_{0}}{2}-1} 2 h_{0}(n) \cos \left(\omega\left(n-\frac{N_{0}-1}{2}\right)\right) & \text { for case } 2\end{cases}
$$

and $h_{0}(n)$ denotes the impulse response of $H_{0}\left(e^{j \omega}\right)$. Similarly, we can express $H_{1}\left(e^{j \omega}\right)$ as [15]

$$
H_{1}\left(e^{j \omega}\right)=j e^{-j \frac{\left(N_{1}-1\right) \omega}{2}} H_{1}(\omega)
$$

where

$$
H_{1}(\omega)=\sum_{n=0}^{\frac{N_{1}}{2}-1} 2 h_{1}(n) \sin \left(\omega\left(n-\frac{N_{1}-1}{2}\right)\right)
$$

and $h_{1}(n)$ denotes the impulse response of $H_{1}\left(e^{j \omega}\right)$. Substituting eqns. 42 and 43 into eqn. 39 yields

$$
\begin{aligned}
T\left(e^{j \omega}\right)= & \frac{1}{L L_{0}} e^{-j\left(G_{0}+N_{0}-1\right) \omega} H_{0}^{2}(\omega) \\
& +\frac{1}{L L_{1}} e^{-j\left(G_{1}+N_{1}-1\right) \omega} H_{1}^{2}(\omega)
\end{aligned}
$$

Let $D_{0}=G_{0}+N_{0}-1$ and $D_{1}=G_{1}+N_{1}-1$. If $D_{0}>$ $D_{1}$, then $\exp \left(-j\left(D_{0}-D_{1}\right) \omega\right)$ must be included in the highpass subband channel, and, if $D_{0}<D_{1}$, then $\exp \left(-j\left(D_{1}-D_{0}\right) \omega\right)$ must be included in the lowpass sub- 
band channel to equalise the group delay difference, and, hence, ensure the PR1 condition. Hence, we can neglect the LP term of eqn. 44 and express $T(\omega)$ as follows:

$$
T(\omega)=\frac{1}{L L_{0}} H_{0}^{2}(\omega)+\frac{1}{L L_{1}} H_{1}^{2}(\omega)
$$

Next, substituting eqns. 42 and 43 into eqns. 40 and 41 , we can obtain

$$
A_{1}(\omega)=\frac{1}{L L_{0}} H_{0}(\omega) H_{0}\left(\omega-\omega_{p}-\omega_{s}\right)
$$

and

$$
+\frac{1}{L L_{1}} H_{1}(\omega) H_{1}\left(\omega-\omega_{p}-\omega_{s}\right)
$$

$$
\begin{aligned}
A_{2}(\omega)= & \frac{1}{L L_{0}} H_{0}(\omega) H_{0}\left(\omega+\omega_{p}+\omega_{s}\right) \\
& +\frac{1}{L L_{1}} H_{1}(\omega) H_{1}\left(\omega+\omega_{p}+\omega_{s}\right)
\end{aligned}
$$

respectively, where the related group delay difference between the lowpass and highpass subband channels is also assumed to be equalised. 